\title{
Speech imagery is not always faster than visual imagery
}

\author{
RITA E. ANDERSON \\ Memorial University of Newfoundland, St. John's, Newfoundland AIB 3X9, Canada
}

\begin{abstract}
One approach to the study of mental imagery is to examine the performance characteristics of different forms of mental imagery when used in various tasks. To demonstrate the utility of this functional approach, the use of speech and visual imagery processes in the serial mental rehearsal of common verbal sequences (e.g., letters of the alphabet) and familiar object arrays (objects found in familiar rooms) was examined in the present experiments. Rehearsal rates and self-reports were consistent with the hypothesis that mental rehearsal efficiency is a function of the compatibility of characteristics of the rehearsal materials and rehearsal mode. While verbal sequences were rehearsed faster under speech than under visual imagery conditions, object arrays were rehearsed as fast under visual as under speech imagery conditions. In addition, evidence was found that covert verbal rehearsal is faster than overt verbal rehearsal under some circumstances.
\end{abstract}

Imagine saying the letters of the alphabet to yourself as rapidly as possible. Now, mentally see the letters of the alphabet, one at a time, as rapidly as possible. Most likely, the first task seemed easier and faster to perform than the second. In fact, results from several experiments show that mentally seeing the letters of the alphabet generally takes two to three times longer than mentally saying the name of each letter (Weber \& Bach, 1969; Weber \& Castleman, 1970). Based on these and other findings, Weber and Castleman (1970) concluded that visual imagery in serial operation is slower than speech imagery and that when one is processing a long serial list, the sequencing of visual images is under verbal control. The latter conclusion was further supported by Weber, Kelley, and Little (1972). They found that the time to classify letters of the alphabet according to their imaged visual characteristics was the same whether subjects said the names of the letters aloud as they visualized them or remained silent and that in both cases, written responses were faster than spoken responses. Thus, even in a visual classification task under visual imagery instructions, subjects appeared to rely on

This research was supported by Grant A6281 from the Natural Sciences and Engineering Research Council of Canada. Portions of these data were presented at the 21 st Annual Meeting of the Psychonomic Society, St. Louis, Missouri, November, 1980. I wish to thank Bruce Fewer for his assistance in collecting and analyzing the data and George Mandler, Mike Rabinowitz, Pat Worden, Bob Weber, and anonymous reviewers for their helpful comments on earlier drafts of the paper. I am also grateful to the hospitality of the Center for Human Information Processing, University of California, San Diego, where this paper was initially written. Address correspondence to Rita E. Anderson, Department of Psychology, Memorial University of Newfoundland, St. John's, Newfoundland A1B 3X9, Canada. implicit verbal rehearsal to facilitate the sequencing of visual images of letters of the alphabet.

Although the evidence appears robust and is subjectively compelling, the conclusions seem problematic on at least two fronts. First, our greater awareness of the effects of task demands and the influence of specific materials on task performance cautions against generalizing from performance on a single task. At the very least, rehearsal of a variety of materials must be examined. Second, the conclusions confer a distinct second-class status on visual imagery as a mode of information processing; they imply that visual imagery processes are subservient to speech imagery processes in the performance of any serial task, and this seems highly improbable. If visual and speech imagery processes are functionally distinct modes of information processing, rather than different types of epiphenomenal noise, the two forms of imagery should be differentially useful in the mental rehearsal of different classes of materials.

As used here, "mental imagery" refers to the variety of processes used to represent and manipulate information in a "mental laboratory" so as to solve problems, answer questions, perform mnemonic functions, formulate thoughts, and so on. Visual and speech imagery processes are viewed as two functionally different modes of mental imagery, which may be used most effectively for different purposes or in different situations. Consider the following examples. When asked "How many window panes are in your living room?" (Meudell, 1971) or "How many windows are in your house?" (Shepard, 1966), people usually claim to visualize the appropriate scene and then count the target items. When trying to determine which letter follows " $t$ " in the alphabet, people frequently hear themselves silently reciting "r-s-t-u." When arranging a room, visual imagery 
is often used to determine which arrangements are plausible. While writing, speech imagery may be used to try out several versions of the idea prior to committing one to paper. These examples suggest a correlation between processing mode and phenomenological content; visual imagery tends to be reported when objects or spatial relations are processed, whereas speech imagery tends to be used when verbal or sequential materials are processed. It appears that while we readily visualize objects and hear inner speech, we rarely engage in inner reading. ${ }^{1}$

This apparent correlation leads to the obvious proposal that speech imagery may be specialized for the processing of verbal/sequential materials and visual imagery may be specialized for the processing of object/pictorial or spatial information (cf. Paivio, 1971). As such, the rehearsal patterns observed by Weber and his colleagues may reflect some aspect of an interaction of processing mode and rehearsal materials, rather than a fundamental difference between speech and visual imagery processes. Implicit verbal rehearsal of letters of the alphabet may be faster than implicit visual rehearsal either because the name and visual/shape codes of letters are differentially accessible to the speech and visual imagery systems, respectively, or because the speech imagery system operates more efficiently with a sequential organization than does the visual imagery system. Since letters of the alphabet are both verbal and sequential, the exact source of the proposed processing compatibility cannot be determined. In contrast, rehearsal of primarily nonverbal, spatially organized stimuli may be facilitated by some form of visual imagery, either because of differential accessibility of the visual/shape and name codes or differential ease of processing of spatial structures by the visual and speech imagery systems, respectively.

To test the compatibility hypothesis using natural stimuli, serial rehearsal of letters of the alphabet and other verbal sequences needs to be compared with serial rehearsal of nonverbal, spatially organized materials. To maximize coding and organizational contrast, the visual/shape codes of items in object arrays should dominate the name codes and the items in the arrays should have a strong spatial organization. Like verbal sequences, the object arrays should consist of a welldefined set in which both the items and their spatial locations are highly overlearned. One set of materials meeting these criteria consists of objects found in specific rooms of a house in which one has been a longtime resident. The items in a given room are defined by their presence and have a regular spatial arrangement, the items and their locations are overlearned through regular use, and they are reacted to in a nonverbal fashion most of the time. If the compatibility hypothesis is valid, rehearsal of objects in rooms should proceed more rapidly with visual than with speech imagery.

Thus far, the problem of sequencing control has been ignored. Sequencing in the rehearsal of letters of the alphabet appears to be under verbal control; does rehearsal of objects in rooms require verbal control as well? Probably not. Paivio (1971) noted that when the response sequence is compatible with the intrinsic structure of the image, the sequencing of visual images can proceed without verbal control. Subjectively, serial rehearsal of objects in rooms seems to be under visual image control. One can start-at a designated spot in the mental room and proceed around it, systematically seeing or naming the objects one by one in spatial sequence. Perhaps rehearsal of object arrays is controlled by the visual imagery system in much the same way that rehearsal of letters of the alphabet is controlled by the speech imagery system.

In summary, the main purpose of the present experiments was to compare the pattern of implicit verbal and visual rehearsal of verbal sequences and object arrays. Prior work had led to claims that in a serial mental rehearsal task, (1) visual imagery is a slower process than speech imagery and (2) the sequencing of visual images is under verbal control. If either or both claims are valid, the rate of implicit visual rehearsal should never equal, much less surpass, the rate of implicit verbal rehearsal for either verbal sequences or object arrays. The alternative presented here suggests that rehearsal rates will be a function of the compatibility of the code or organizational structure of the rehearsal materials and the rehearsal mode. Thus, object arrays are expected to be rehearsed more rapidly by the visual imagery than by the speech imagery system; for verbal sequences, the reverse should be observed.

At this point, it should be noted that object arrays and verbal sequences are not comparable stimulus sets. For instance, a couch in the living room is vastly different from the letter $\mathrm{C}$ whether referred to by visual or speech imagery processes. A visual image of a couch, whether visualized skeletally or in fully articulated detail, is likely to be informationally denser than a visual image of the letter $\mathrm{C}$, and hence it might take longer to generate a visual image of an object than of a letter. In addition, name codes for objects are probably more variable than name codes for verbal items, thus affecting naming times. These considerations suggest that direct comparisons of performance on object arrays and verbal sequences are difficult to interpret. The strategy here, as elsewhere (see Anderson, 1976; Smith \& Magee, 1980), is to concentrate on qualitative, as opposed to quantitative, effects. The pattern of speech and visual imagery rehearsal of object arrays will be compared with the pattern of rehearsal of verbal sequences. Since different processes may be reflected in the rates of implicit verbal and visual rehearsal, this approach does not necessarily eliminate all interpretive difficulties. Further consideration of these problems, however, will be deferred to the final discussion.

A second purpose of the present experiments was to determine whether the twofold superiority of implicit verbal over visual rehearsal of letters of the alphabet 
would generalize to other overlearned verbal sequences, such as days of the week and months of the year. Since individual items in the latter sequences are larger than those in the alphabet (e.g., whole words vs. single letters), both visual and speech imagery rehearsal rates (e.g., items per second) will be reduced. If the two rates decrease proportionately, the twofold advantage of implicit verbal rehearsal will be maintained. To the extent that the visual system can successfully generate several letters in parallel (cf. Weber \& Harnish, 1974; Weber et al., 1972), the decrement in the rate of implicit visual rehearsal may be less than the decrement in the rate of implicit verbal rehearsal. If so, the advantage of implicit verbal rehearsal may be substantially reduced when word sequences are rehearsed.

\section{EXPERIMENT 1}

\section{Method}

Subjects. Subjects were 10 males and 10 females from the subject pool at Memorial University of Newfoundland who were paid for their services.

Materials. Seven rehearsal sets were used. Verbal sets included the following sequences: letters of the alphabet, days of the week, and months of the year. Object arrays consisted of items found in three different target rooms from the subject's family home: the living room, the kitchen, and the subject's own bedroom. A seventh rehearsal set, which was neither clearly verbal nor clearly nonverbal, consisted of immediate family members. Verbal labels for each of the seven rehearsal sets were typed and photographed for slide presentation.

Design and Procedure. Rehearsal mode (implicit verbal and visual), target class (verbal sequences and object arrays), and test blocks (1-12) were within-subjects factors. Rehearsal mode was alternated across test blocks; half of the subjects began with implicit verbal rehearsal, and the remainder began with implicit visual rehearsal. Hence, each subject was tested for six test blocks in each rehearsal mode.

Subjects were told that the purpose of the experiment was to determine the rate at which people could mentally rehearse items from a variety of rehearsal sets. For implicit verbal rehearsal, subjects were instructed to say the names of the items in the different rehearsal sets to themselves; for implicit visual rehearsal, they were instructed to mentally see the items in the different rehearsal sets, one item at a time. In all cases, subjects were told to rehearse at a fast, but personally comfortable, rate.

For verbal sequences, subjects were told to start at the beginning of the target sequence and to continue through to the end, carefully seeing or saying each item in the sequence (e.g., each letter in the alphabet one at a time, each day of the week, one at a time, etc.). For object arrays, subjects were told to use rooms from their family homes, or the place where they had spent the most time while growing up. They were instructed to start at one point in the target room and carefully name or see, one at a time, "the items or objects which are characteristic of the target room in your home. Mentally see [name] those items which are almost always observable or present in the target room and which are important to your conception of that room at home." Subjects practiced using both rehearsal modes on the digits 1.10 and objects found in their bathroom at home. After each practice trial, subjects described how they had performed the task. Once both experimenter and subject felt that the task requirements had been understood, the experimental session began.

Each of the 12 test blocks consisted of one rehearsal trial on each of the rehearsal sets. At the beginning of each test block, the subject was told to use either implicit verbal or visual rehearsal to rehearse all sets in that block. The appropriate cue card, containing either the word SEE or the word SAY, was visible to the subject throughout each test block. Subjects were instructed to rehearse the same items in each rehearsal set in the same order across the 12 test blocks.

The procedure for each test trial was as follows. The subject was seated before a small screen on the wall, with a response button beside him or her. At the beginning of each trial, the experimenter said, "Ready." One second later, the experimenter pressed a button that simultaneously started the Hunter digital timer and opened the Gerbrands shutter on the Kodak Carousel slide projector, thereby exposing the name of the rehearsal set. As soon as the subject completed rehearsal of the items, he or she pressed the response button. This terminated the display and stopped the timer. The experimenter recorded the time, reset the timer, and advanced the slide projector. After $5 \mathrm{sec}$, a new trial was initiated. No feedback concerning rehearsal latencies was provided.

In each of the first six test blocks, the three verbal sequences were followed by the immediate family set and then by the three object arrays. In the final six test blocks, this order was reversed. The position of specific verbal and object sets in a test block varied across test blocks so that each set was tested once in Positions 1-3 and 5-7 under each rehearsal mode. Half of the subjects who began with implicit verbal (visual) rehearsal were tested on Test Blocks 1-12 as described above; the other half were tested in the reverse order (e.g., Test Blocks 12-1).

After the 12th test block, the accuracy of rehearsal of verbal sequences and the composition of the object arrays was assessed. Rehearsal sets were presented as before, and subjects were asked to name the members of each set aloud, at a slow rate so that the experimenter could record them. Subjects were instructed to say the items in the order in which they had rehearsed them and to use the names they had used in the rehearsal test blocks.

Following the overt naming trial, subjects completed a pencil-and-paper questionnaire designed to assess three aspects of subjective task experience. The first question involved perceived task difficulty. For this question, subjects had to choose which of two tasks seemed easier to perform. The four different task pairings in which either rehearsal mode or target class differed, but not both, were tested. For instance, task pairings such as saying sequences and seeing sequences were tested, but task pairings such as saying sequences and seeing objects were not. The second question assessed subjective use of verbal and visual/shape codes while performing the task. For each of the rehearsal mode by target class conditions, subjects checked the statement that best described the coding format of their subjective experience. The third question was designed to assess the qualitative nature of the verbal and visual images in the different tasks; subjects were asked to check the statement that best described the quality of their imagery in the different tasks. The wording of the statements for the second and third questions is shown in Tables 1 and 2 .

Table 1

Number of Subjects Who Selected Each Statement as the Best Description of How They Performed Each Task

\begin{tabular}{lrrrr} 
& \multicolumn{3}{c}{ Conditions } \\
\cline { 2 - 5 } \multicolumn{1}{c}{ Statement } & $\begin{array}{c}\text { Verbal } \\
\text { Sequences }\end{array}$ & \multicolumn{1}{c}{$\begin{array}{c}\text { Object } \\
\text { Arrays }\end{array}$} \\
\cline { 2 - 5 } \cline { 3 - 5 } & Saying & Seeing & Saying & Seeing \\
\hline I only named them & 12 & 2 & 0 & 0 \\
I named then visualized them & 1 & 6 & 3 & 0 \\
I visualized then named them & 5 & 7 & 17 & 5 \\
I only visualized them & 0 & 4 & 0 & 14 \\
Other & 2 & 1 & 0 & 1 \\
\hline
\end{tabular}

Note-Maximum $=20$. 
Table 2

Number of Subjects Who Selected Each Statement as the Best Description of Their Imaginal Experience While Performing the Task

\begin{tabular}{lcc}
\hline \multicolumn{1}{c}{ Description } & \multicolumn{2}{c}{ Target Class } \\
\cline { 2 - 3 } "Saying" Rehearsal Mode & $\begin{array}{c}\text { Verbal } \\
\text { Sequences }\end{array}$ & $\begin{array}{c}\text { Object } \\
\text { Arrays }\end{array}$ \\
\hline Full name, well pronounced & 15 & \\
Full name, slurred pronunciation & 3 & 7 \\
Partial name, well pronounced & 1 & 1 \\
Partial name, slurred pronunciation & 0 & 4 \\
Other & 1 & 0 \\
\multicolumn{1}{c}{ "Seeing" Rehearsal Mode } & & \\
Clear, detailed, close-up image & 5 & 10 \\
Clear, detailed, distant image & 4 & 6 \\
Fuzzy, close-up image & 4 & 1 \\
Fuzzy, distant image & 4 & 1 \\
Other & 3 & 2 \\
\hline
\end{tabular}

\section{Results and Discussion}

Rehearsal data. Data were analyzed both in terms of rehearsal rate (number of items rehearsed/total rehearsal time) and rehearsal time per item (total rehearsal time/ number of items rehearsed). Because extreme latencies are frequently more disruptive in a time-based than in a rate-based analysis, only the results of the rate analyses will be presented. In fact, both analyses led to the same conclusions. Unless otherwise noted, all effects reported throughout this paper were reliable at the .01 level of significance or beyond.

Rehearsal rates for each of the six experimental rehearsal sets in each test block were calculated for each subject. Since all subjects correctly recited the verbal sequences on the final overt test block, set size for these sequences was assumed to be the number of items in each sequence. Set size for object arrays was assumed to be equal to the number of items emitted for each target room in the final overt test block.

To assess the predicted Target Class by Rehearsal Mode interaction, the mean rehearsal rate for verbal sequences and for object arrays in each test block was calculated for each subject from his or her individual rehearsal rates for the three verbal and object rehearsal sets. These means were entered into an analysis of variance in which rehearsal mode (implicit verbal and visual), target class (verbal sequences and object arrays), and functional test block (1-6) were within-subjects factors. Functional Test Blocks 1-6 refer to the first through sixth uses of a given rehearsal mode. While the use of functional test blocks facilitated analysis, it had the consequence of including, for example, in Functional Test Block 1, data from subjects whose first experience with a given rehearsal mode occurred on the first or second experimental test block. Since rehearsal patterns of the two subgroups of subjects were very similar, the simplification gained by the use of functional, rather than nominal, test block seems justified.
In this analysis, rehearsal mode, target class, and their interactions $[\mathrm{Fs}(1,19)=52.55,50.30$, and 45.66 , MSes $=.85,4.13$, and 1.61 , respectively $]$ were all significant. As shown in Figure 1,implicit verbal rehearsal was substantially faster than implicit visual rehearsal for rehearsal of verbal sequences. For object arrays, the trend was in the opposite direction, with implicit visual rehearsal rates being consistently higher than implicit verbal rehearsal rates. Rehearsal rates increased across test blocks $[\mathrm{F}(5,95)=18.06, \mathrm{MSe}=.16]$, and as the triple interaction shown in Figure 1 reveals, the practice effect was greatest for implicit verbal rehearsal of verbal sequences $[\mathrm{F}(5,95)=3.53, \mathrm{MSe}=.08]$.

Separate analyses were performed on data from the verbal sequence and object array rehearsal sets to determine the contribution of specific rehearsal sets to the overall pattern. In these analyses, each subject's rehearsal rate for the individual rehearsal sets was entered into separate analyses of variance in which rehearsal mode (implicit verbal and visual), rehearsal set (the appropriate three verbal sequence or object array sets), and functional test block (1.6) were within-subjects factors. The results of these analyses nicely confirmed the major patterns noted above.

Verbal sequences. Rehearsal rates for verbal sequences are shown as a function of test block in Figure 2. There, it can be seen that letters of the alphabet were rehearsed faster than the other verbal sequences $[F(2,38)=60.68$, $\mathrm{MSe}=5.07]$, that implicit verbal rehearsal was faster than implicit visual rehearsal $[\mathrm{F}(1,19)=76.74, \mathrm{MSe}=$ $4.54]$, and that the difference between implicit verbal and visual rehearsal rates was greater for letters of the alphabet than for either of the other sequences $[F(2,38)$ $=45.99$, MSe $=1.28]$. Test block was significant as a main effect and in interaction with all other factors. The triple interaction shown in Figure 2 shows that

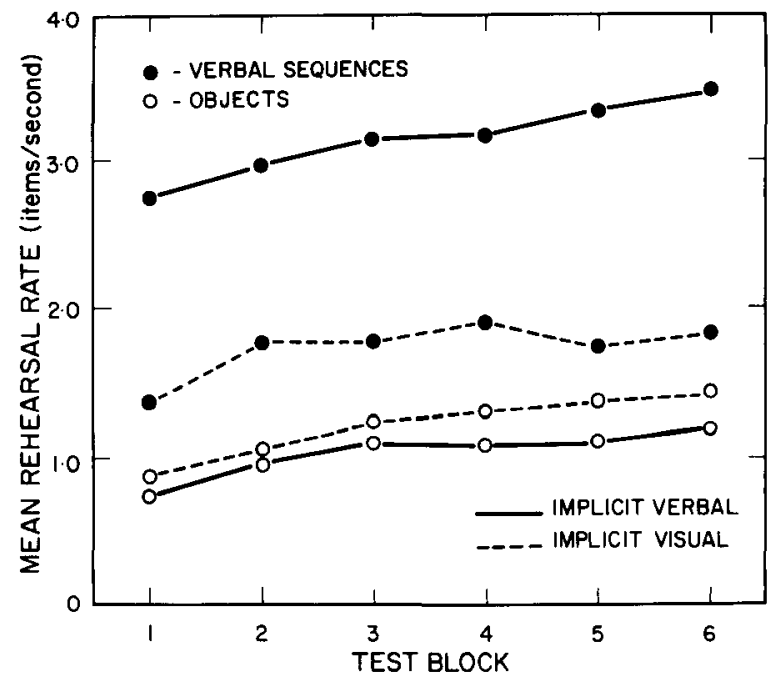

Figure 1. Mean rehearsal rates from Experiment 1 for object arrays and verbal sequences as a function of functional test block. 


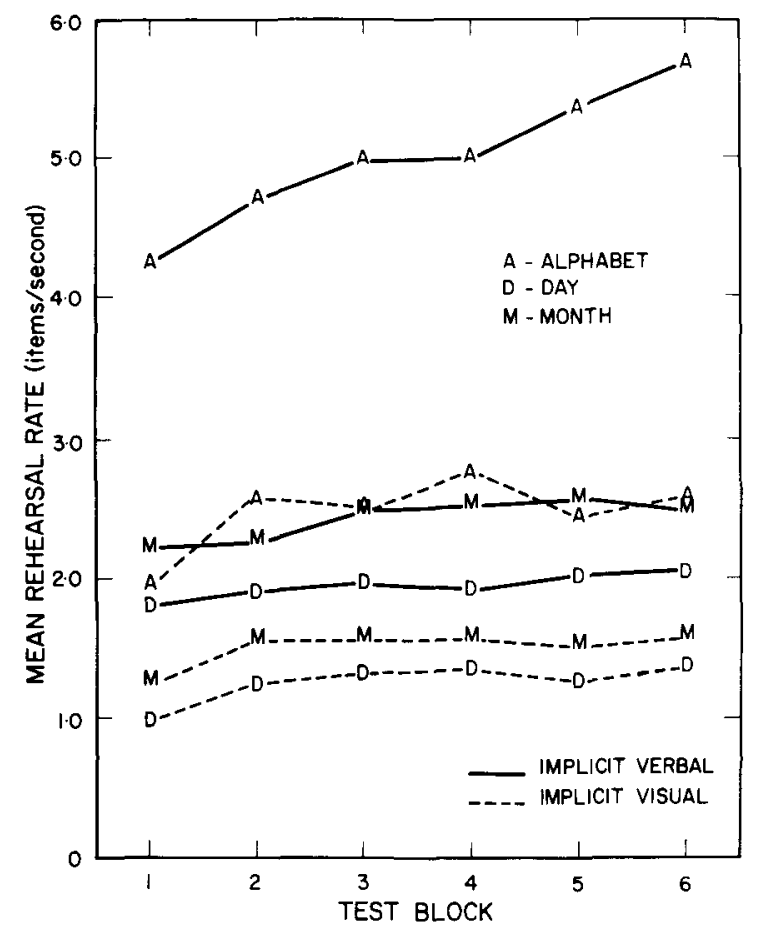

Figure 2. Mean rehearsal rates from Experiment 1 for the three specific verbal sequences as a function of functional test block.

practice effects were most pronounced for implicit verbal rehearsal of letters of the alphabet $[F(10,190)=$ $2.18, \mathrm{MSe}=.18, \mathrm{p}<.05]$.

The observed interaction of rehearsal mode and rehearsal set would seem to imply that the advantage of implicit verbal over visual rehearsal is not a constant but varies according to the nature of the verbal sequence. However, the use of difference scores by themselves may be misleading, since both the day and month sequences were rehearsed at a slower unit rate than were letters. Although there is no acceptable way to calculate rehearsal rates for the different sequences using the same unit size (e.g., phonemes cannot be used because of the problem of phoneme dropping in rapid speech), ratio comparisons (e.g., rate of implicit verbal rehearsal/ rate of implicit visual rehearsal) should be independent of unit-size differences. This ratio was calculated for each subject for each of the verbal sequences. For letters of the alphabet, implicit verbal rehearsal was 2.52 times faster than implicit visual rehearsal; for the month and day sequences, the respective values were 1.85 and 1.77 . The month and day ratios were smaller than the alphabet ratio for 18 and 17 of the 20 subjects, respectively. These analyses suggest that while verbal sequences are generally rehearsed most rapidly by the speech imagery system, the size of the advantage varies according to the nature of the sequence. Perhaps the reduction of the superiority of implicit verbal rehearsal for the day and month sequences reflects the ability of the visual imagery system to process several letters in parallel (Weber \& Harnish, 1974).
Object arrays. In the analysis of object arrays, rehearsal mode was not significant as a main effect $[F(1,19)=$ $1.94]$, but it did interact with rehearsal set $[F(2,38)=$ $3.78, \mathrm{MSe}=.08, \mathrm{p}<.05]$. In addition to being greater than implicit verbal rehearsal, the rate of implicit visual rehearsal varied across target rooms $(1.13,1.24$, and $1.26 \mathrm{objects} / \mathrm{sec}$ for the kitchen, bedroom, and living room, respectively), whereas the rate of implicit verbal rehearsal remained constant across target rooms (1.03$1.05 \mathrm{objects} / \mathrm{sec}$ ). The only other significant effect was the expected practice effect $[\mathrm{F}(5,95)=14.85, \mathrm{MSe}=$ .27].

More items were generated for the living room than for the kitchen or bedroom rehearsal sets $(9.1$ vs. 7.5 and 7.9 items, respectively). And, of course, the number of items in each object array varied widely across rooms and subjects, ranging from 2 to 17 objects. However, there was no evidence that array size had any systematic effect on rehearsal rates, in that rehearsal rates for small and large arrays were equivalent.

Questionnaire data. As predicted, the rate of implicit visual rehearsal of object arrays was greater than the rate of implicit verbal rehearsal, but the effect was small in comparison with the effects observed for rehearsal of verbal sequences. In contrast, the questionnaire data were decisive. In the question concerning task difficulty, $85 \%$ of the total choices conformed to expectation. Subjects claimed that "saying the names of words or letters in sequence" was easier than "saying the names of objects in the room" (95\%) and easier than "seeing words or letters in sequence" (75\%). In addition, subjects claimed that "seeing objects in the rooms" was easier than "seeing words or letters in sequence" $(85 \%)$ and easier than "saying the names of objects in the rooms" $(85 \%)$. Table 1 shows the number of subjects who chose each statement as the best description of how they performed each task. With the exception of the task of seeing verbal sequences, their choices were remarkably close to expectations. The responses shown in Table 2 suggest that the highest quality imagery tends to occur in conditions in which rehearsal mode is compatible with target class. Thus, for implicit verbal rehearsal, $75 \%$ of the subjects claimed to clearly pronounce the full name of the words and letters, but only $40 \%$ made the same claim for pronunciation of names of objects. For implicit visual rehearsal, $50 \%$ of the subjects claimed that their visual images of objects were detailed, clear, and close-up; only $25 \%$ made the same claim for visual images of letters and words.

Subjects were asked when they had last visited the rooms in the house that they used for the rehearsal task. Only one subject had not been home within the last month, and the median absence was 2 weeks.

\section{EXPERIMENTS 2 AND 3}

Although the results of Experiment 1 appear straightforward, the questionnaire data raise the specter that the rehearsal patterns reflect subject expectations rather 
than what normally might occur. To reduce the transparency of the experimental question, two experiments were performed in which rehearsal mode was a betweensubjects factor. Different item selection instructions for object arrays were used in the two experiments to determine if specific item selection instructions affected the pattern of rehearsal of object arrays.

\section{Method}

Subjects. The 48 paid subjects, 24 in each experiment, were obtained from the subject pool. Within experiments, subjects were assigned to rehearsal conditions according to a prearranged random order upon their arrival at the laboratory.

Design and Procedure. The design and procedure were identical to those of Experiment 1, with the following exceptions. First, rehearsal mode (implicit verbal or visual) was a between- rather than a within-subjects factor. Second, the number of test blocks was reduced to three. Finally, item selection instructions for object arrays were modified slightly. In Experiment 2, item selection instructions were based on those used in Experiment 1, but in Experiment 3, item selection instructions were more restrictive. Subjects were instructed to mentally name or see "only major pieces of furniture or appliances, such as objects which take up a fair amount of space and sit on the floor."

Each subject was tested for three test blocks and then overtly named the items in each rehearsal set, as before. Half of the subjects in each condition were tested on the verbal sequences first, the immediate family set next, and finally, on the object arrays. The other half were tested in the reverse order. Within the verbal sequence and object array subblocks, each rehearsal set was tested first, second, and third equally often. Thus each subject was tested on a unique ordering of rehearsal sets that remained constant across the three test blocks.

\section{Results and Discussion}

The rehearsal data from each experiment were analyzed separately and together, with experiments treated as a between-subjects factor. Since the results of the combined analysis were consistent with those of the separate analysis and are most informative, only the results of the combined analyses will be presented.

Different item selection instructions affected the size of the object arrays. Larger rehearsal sets were generated in Experiment 2 (8.2 objects) than in Experiment 3 (5.7 objects) $[\mathrm{F}(1,44)=18.16, \mathrm{MSe}=12.25]$. Although the size of the object arrays varied across experiments, rehearsal rates were not affected; in all analyses, experiment failed to approach significance, either as a main effect or in interaction with any. other variable.

Rehearsal rates for verbal sequences and object arrays are shown in the left panel of Figure 3, and rehearsal rates for the three different verbal sequences are shown in the right panel. In both cases, the rehearsal patterns replicated those of Experiment 1 quite closely.

In the overall analysis, rehearsal mode, target class, and their interaction were significant $[\mathrm{Fs}(1,44)=12.53$, 191.34 , and 21.20 , MSes $=1.84$ and .98 , respectively $]$. Object arrays were rehearsed at the same rate regardless of rehearsal mode, whereas implicit verbal rehearsal of verbal sequences was substantially faster than implicit

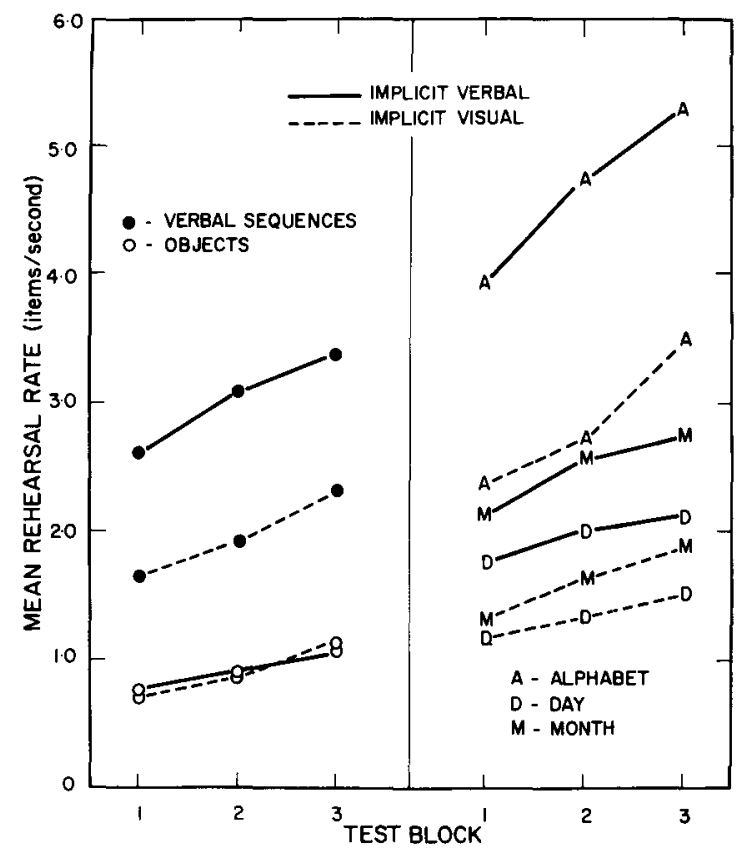

Figure 3. Mean rehearsal rates from Experiments 2 and 3 as a function of test block for object arrays and verbal sequences (left panel) and for the three specific verbal sequences (right panel).

visual rehearsal. Rehearsal rates increased as a function of test blocks $[F(2,88)=71.80, \mathrm{MSe}=.11]$, especially for verbal sequences, as revealed by the significant Test Block by Target Class interaction $[\mathrm{F}(2,88)=8.80, \mathrm{MSe}=.07]$.

In the analysis of verbal sequences, rehearsal rates were highest for letters of the alphabet and lowest for days of the week $[\mathrm{F}(1,88)=142.84, \mathrm{MSe}=1.30]$. Implicit verbal rehearsal was faster than implicit visual rehearsal $[\mathrm{F}(1,44)=18.30, \mathrm{MSe}=7.17]$, and the rate difference between implicit verbal and visual rehearsal was greatest for letters of the alphabet $[F(2,88)=11.13$, $\mathrm{MSe}=1.30]$. Finally, the main effect of test block $[\mathrm{F}(2,88)=70.97, \mathrm{MSe}=.27]$ was qualified by the significant interaction of Rehearsal Set by Test Block $[F(4,176)=19.93, \mathrm{MSe}=.14]$. In the analysis of object arrays, the only effect to achieve significance was test block $[F(2,88)=22.09, \mathrm{MSe}=.28]$.

The consistency of the patterns of rehearsal rates across Experiments 1-3 implies that rehearsal speed is not a constant but is a function of the compatibility of characteristics of the rehearsal mode and rehearsal materials. Under most circumstances, for verbal sequences, implicit verbal rehearsal will be faster and will seem easier than implicit visual rehearsal. For object arrays, implicit visual rehearsal will either be faster than or equal to implicit verbal rehearsal and will seem easier than implicit verbal rehearsal. Before these results can be interpreted, a control problem needs to be addressed.

It has been assumed that during the experimental test blocks subjects mentally rehearse the items that they name during the final overt naming trial. Specifi- 
cally, it has been assumed that the composition of rehearsal sets remains constant across trials, and that the same items in each rehearsal set are rehearsed in the same order across the test blocks. Most likely, these assumptions are valid for rehearsal of verbal sequences, since the sequences are extremely well-defined and overlearned. But what of rehearsal of object arrays? Although they are overlearned, both the items and the order of rehearsal are determined by the subject. Unless the above assumptions can be shown to hold for rehearsal of object arrays, the present results become less convincing. One way to assess these assumptions would be to force subjects to use some form of overt rehearsal. While it is difficult to create a satisfactory overt equivalent of implicit visual rehearsal, subjects can engage in overt verbal rehearsal. The items included in each overt rehearsal of a given rehearsal set can then be compared with those included in a final "overt naming" trial to assess the consistency of rehearsal sets across trials.

\section{EXPERIMENTS 4 AND 5}

To mimic the testing conditions of Experiment 1, subjects alternated across test blocks between overt and covert verbal rehearsal of the items in previously used rehearsal sets. This design also allowed for a test of the relative speed of overt and covert rehearsal. Conventional wisdom has it that overt and covert rehearsal occur at the same rate and involve many of the same central processes (Dell, 1977; Landauer, 1962; Weber \& Bach, 1969). As it turned out, covert rehearsal was significantly faster than overt rehearsal in Experiment 4. One major difference between Experiment 4 and prior work involved the use of a tape recorder. Most subjects in Experiment 4 expressed fear or embarrassment at being taped. Since people occasionally "choke-up" while being recorded, it seemed that failure to replicate conventional wisdom was due, in part, to the use of a tape recorder. Hence, in Experiment 5, subjects were not taped while they performed the task.

\section{Method \\ The 12 paid subjects in each experiment were recruited from the subject pool at Memorial University. With the excep- tion of the change in rehearsal mode, instructions relevant to that change, and the use of a tape recorder in Experiment 4, the design and procedures for both experiments were identical to those used in Experiment 1. In both Experiments 4 and 5, speech mode referred to the use of overt and covert verbal rehearsal; subjects were instructed to alternate across test blocks between rehearsing the names of the items in the rehearsal sets aloud and to themselves. In Experiment 4, a Sony TC-110B cassette tape recorder was placed on the table to the right of the subject. It was turned on at the beginning of the first block and allowed to run continuously through the final overt naming block. No tape recorder was present in Experiment 5 .}

\section{Results and Discussion}

Experiment 4: Rehearsal sets. To determine the extent to which the final overt naming trial reflected the composition of the rehearsal sets across test blocks, the composition of each rehearsal set in each overt rehearsal trial was compared with the composition of the appropriate rehearsal set on the 13 th overt naming trial. For each subject, the absolute number of deviations, including both additions and deletions, from the set of items named in the 13th overt trial was recorded for each comparison. The mean absolute deviation for object arrays was somewhat larger for the living room than for the kitchen or bedroom rehearsal sets $(.52, .29$, and .25 objects, respectively). For all rooms, the mean absolute deviation decreased across Test Blocks 1.6 (1.19, .36, $.14, .19, .11$, and .11 objects, respectively).

To assess the consistency of order of rehearsal across test blocks, the rehearsal order of the items in each overt trial was compared with the output order in the 13th overt trial using a bidirectional measure of intertrial repetition, ITR(2) (Mandler \& Dean, 1969). This measure yields a number from .00 to 1.00 , where .00 represents no correspondence between the two orders and 1.00 indicates perfect correspondence. The ITR(2) values varied from .86 to .82 across rooms and increased monotonically across Test Blocks $1-6(.70, .78, .85$, $.87, .88$, and .93 , respectively). For comparison purposes, reversal of only the last two items in a sequence would yield ITR(2) values of $.50, .67, .75$, and .80 for sequence lengths of three, four, five, and six items, respectively. The obtained $\operatorname{ITR}(2)$ values reveal that subjects rarely made reversal errors and that they were remarkably adept at rehearsing the same items in the same order across test blocks. No statistics were calculated for rehearsal of verbal sequences, since subjects consistently rehearsed the proper items in the correct order on all but a few occasions.

Experiments 4 and 5: Rehearsal rates. Rehearsal rates were analyzed for each experiment separately, using the analysis of variance designs described for Experiment 1, and together with experiments as a between-subjects factor. Since the combined analyses were most informative and were consistent with the other set of analyses, only the combined analyses will be presented. In these analyses, experiments failed to approach significance, either as a main effect or in interaction with any other factor. Hence, the overall pattern of rehearsal rates apparently was not predicated upon the presence or absence of a tape recorder, as had been suggested.

In the overall analysis, target class and speech mode were significant as main effects $[\mathrm{Fs}(1,22)=240.91$ and 34.58 , MSes $=2.37$ and .93 , respectively $]$ and in interaction with each other $[\mathrm{F}(1,22)=6.77, \mathrm{MSe}=.44$, $\mathrm{p}<.025]$. As shown in Table 3, verbal sequences were rehearsed faster than object arrays, and covert rehearsal was faster than overt rehearsal. In addition, the difference between covert and overt rehearsal rates was greater for rehearsal of verbal sequences than for object arrays. If the ratio analysis is used to compensate for the difference in base rehearsal rates, a different picture emerges. That is, covert rehearsal was 1.39 times faster 
Table 3

Mean Rehearsal Rates for Overt and Covert Rehearsal of Different Rehearsal Sets From the Analyses of Experiments 4 and 5

\begin{tabular}{lccc}
\hline & \multicolumn{3}{c}{ Speech Mode } \\
\cline { 2 - 4 } \multicolumn{1}{c}{ Rehearsal Set } & Overt & Covert & Mean \\
\hline Letters of the Alphabet & 4.20 & 5.38 & 4.79 \\
Days of the Month & 1.84 & 2.07 & 1.96 \\
Months of the Year & 2.13 & 2.58 & 2.35 \\
Mean: Verbal Sequences & 2.72 & 3.34 & 3.03 \\
Mean: Object Arrays & .88 & 1.21 & 1.04 \\
\hline
\end{tabular}

than overt rehearsal of object arrays and only 1.20 times faster than overt rehearsal of verbal sequences. Rehearsal rates also increased with test block $[\mathrm{F}(5,110)$ $=47.34, \mathrm{MSe}=.08]$.

In the analysis of verbal sequences, speech mode $[F(1,22)=31.09, \mathrm{MSe}=2.64]$, rehearsal set $[\mathrm{F}(2,44)=$ $153.40, \mathrm{MSe}=4.40]$, and their interaction $[\mathrm{F}(2,44)=$ $30.53, \mathrm{MSe}=.58]$ were significant. As shown in Table 3, letters of the alphabet were rehearsed faster than word sequences overall; in addition, the difference between covert and overt rehearsal rates was greatest for letters of the alphabet. The ratio analysis confirmed this interaction. Rehearsal rates increased with test block $[\mathrm{F}(5,110)=22.50, \mathrm{MSe}=.29]$, and the interaction of Rehearsal Set by Test Block $[\mathrm{F}(10,220)=5.93$, MSe $=$ .19] revealed that practice effects were most pronounced for rehearsal of letters of the alphabet. In the analysis of object arrays, covert rehearsal was faster than overt rehearsal $[F(1,22)=15.92, \mathrm{MSe}=1.47]$. In addition, while rehearsal rates increased with test block $[\mathrm{F}(5,110)=40.63, \mathrm{MSe}=.12]$, the practice effect was more pronounced for covert than for overt rehearsal $[\mathrm{F}(5,110)=3.39, \mathrm{MSe}=.08]$.

In the present experiments, covert rehearsal was consistently faster than overt rehearsal. MacKay (in press) also has found that subjects can mentally say sentences faster than they can overtly say them. No unambiguous reasons can be proposed to account for these failures to replicate previous findings of no difference. One possibility might involve the contrast between instructions in the present experiments to rehearse at a "fast, but personally comfortable, rate" and previous instructions to rehearse as rapidly as possible. However, MacKay instructed his subjects to rehearse as fast as they could and still found covert speech to be faster than overt speech. Another possibility might involve amount of practice. Landauer (1962) claimed that, initially, covert speech tended to be faster than overt speech and only with practice did the two rates converge. No evidence of convergence was found in the present data; the only interaction of rehearsal mode and test block was in the opposite direction. And, although MacKay's data show a tendency toward eventual convergence, even after 12 covert or overt trials, covert speech was still considerably faster than overt speech. While further work is obviously needed to clarify the conditions that determine the relative rates of covert and overt speech, it seems reasonable that thinking about speaking without activating the motoric commands might, under some circumstances, be faster than actually speaking.

\section{GENERAL DISCUSSION}

Since the results of Experiment 4 demonstrated that subjects could, and probably did, rehearse the same items in the same order across test blocks, it is appropriate to review and discuss the major findings of Experiments 1-3. First, the basic findings reported by Weber and his colleagues (e.g., Weber \& Bach, 1969; Weber et al., 1972) were clearly replicated. Implicit verbal rehearsal of letters of the alphabet was two to three times faster than implicit visual rehearsal. Second, the pattern of implicit verbal and visual rehearsal rates varied substantially as a function of target class. For verbal sequences, implicit verbal rehearsal rates were consistently greater than implicit visual rehearsal rates; for object arrays, the rate of implicit verbal rehearsal was either slightly less than or equal to the rate of implicit visual rehearsal. In addition, subjects in Experiment 1 reported that implicit verbal rehearsal was easier than implicit visual rehearsal for rehearsing verbal arrays and vice versa for rehearsing object arrays. Third, relative rehearsal rates within verbal sequences varied in that the advantage of implicit verbal over implicit visual rehearsal was greater for letters of the alphabet than for either the day or the month sequences. These effects were obtained whether rehearsal mode was a betweenor within-subjects variable.

The above findings refute the generality of both the hypothesis that visual imagery in serial operation is slower than speech imagery and the hypothesis that the sequencing of visual images is under verbal control. Had either of these hypotheses been generally valid, the rate of implicit visual rehearsal of object arrays could not have equaled the rate of implicit verbal rehearsal. Nor would implicit visual rehearsal have been deemed easier than implicit verbal rehearsal for rehearsal of object arrays. Instead, it appears that rehearsal may be faster and easier to perform when the processing mode is compatible with the characteristics of the rehearsal materials than when the two are incompatible.

The question arises as to whether the various rehearsal rates reflect the operation of similar processes. First, consider rehearsal of object arrays and verbal sequences. At the most global level, similar processes are involved. To rehearse either type of target, the subject must activate and retrieve the relevant set from memory and then process each item in the set serially. However, as noted in the introduction, verbal sequences and object arrays are not strictly comparable. If any of the differ- 
ences between the two target classes affect how the mental rehearsal task is performed, rehearsal rates for verbal sequences may reflect processes different from those reflected by rehearsal rates for object arrays.

One difference is that the composition, number, and sequencing of the items is absolutely determined a priori for verbal sequences, whereas for object arrays, all selection is under subject control. As a result, rehearsal rates for object arrays reflect item selection and sequencing times, as well as item rehearsal times. Since fairly stable rehearsal sets appear to be established within a few trials, the impact of item selection and sequencing decisions should be minimal in later test blocks. Another difference is that objects can be named and imaged in a greater variety of ways than can verbal items; thus, rehearsal rates for object arrays in the initial test blocks will reflect additional time taken by subjects to decide which representation to generate. These differences point to the necessity of restricting interpretation to within-class comparisons. That way, the "contamination" of item selection, sequencing, and representation decision times will be restricted to rehearsal of object arrays.

Do implicit verbal and visual rehearsal rates reflect the operation of comparable processes? Although both require subjects to process items serially, the two rehearsal modes can be performed differently. That is, while implicit verbal rehearsal can be performed in only one way (e.g., serial generation of the target verbal code), implicit visual rehearsal can be performed in at least two ways. Subjects can generate visual images of single target items, one by one, in a manner strictly comparable to implicit verbal rehearsal. Alternatively, subjects can generate a holistic image of the target set and then scan and/or zoom in on each of the items in the holistic array. For instance, they could generate an image of the entire alphabet, as in a grade school display, or of the entire target room and then inspect each item in the array one by one.

Intuitively, holistic generation plus scanning seems most likely to occur in the rehearsal of object arrays. How often it was used is open to question. Although subjects were instructed to rehearse items, one by one, there is no guarantee that they generated them, rather than scanned them. Aside from instructions, the only other factor constraining use of the generation-plusscanning strategy is the limited capacity of the visual image system (Kosslyn, 1980). In Experiment 1, 80\% of the subjects reported generating clear and detailed images of the objects. Given the capacity limits of the system, it seems unlikely that subjects could maintain and scan a fully articulated image of a room plus all of its contents. It is possible, however, that subjects may have engaged in a combination strategy of holistic skeletal generation plus scanning and/or zooming in on and elaborating the details of target items.

The above raises yet another issue. Objects can be imaged skeletally or in fully articulated detail. Since image generation time increases as degree of elaboration increases (Kosslyn, 1980), the degree of elaboration will affect the rate of implicit visual rehearsal of object arrays. As noted above, subjects tended to report clear, detailed images; had these subjects generated skeletal images, implicit visual rehearsal rates might have been higher. Note that this type of problem is not restricted to implicit visual rehearsal of object arrays. As indicated by the questionnaire data, images of verbal units may not always be well formed; furthermore, names of objects or verbal units may not always be clearly and fully pronounced. Further work is clearly needed to determine the effects of generation vs. scanning of elaborate or skeletal images on the rate of implicit visual rehearsal. Also, the influence of the quality of implicit speech on rehearsal rates needs further investigation.

The above discussion indicates that the obtained rehearsal rates may have been influenced by a variety of factors. As such, the rates cannot be interpreted further or compared in any absolute sense. The rehearsal rates simply reflect the speed at which subjects can serially process items from different target classes using subject-determined speech and visual imagery processes. At the very least, the present results lend support to the compatibility hypothesis. They also suggest that if a classification task similar to that used by Coltheart, Hull, and Slater (1975) were used to control the quality of the imaginal code and to ensure that items were clearly processed one by one, the compatibility hypothesis would be confirmed.

Since organizational structure and dominant code were confounded in the rehearsal materials used in these experiments, the source of the proposed compatibility cannot be identified. Further work using artificially learned rehearsal sets in which organizational structure and coding have been experimentally manipulated is required to determine the source of the compatibility.

While the present experiments have raised more questions than they have answered, they will have served their purpose if they encourage more research on the functional uses of different forms of mental imagery (see also Kolers \& Smythe, 1979). Although much has been learned about the operating characteristics of visual imagery by studying it directly, additional valuable information can be gained by comparing the functional utility of visual imagery with other forms of mental imagery. As the present data show, different forms of mental imagery function most usefully on different types of information. Even the "difficulties" noted above are important, for they point to the flexibility of the two systems of mental rehearsal.

\section{REFERENCES}

Anderson, R. E. Short-term retention of the where and when of pictures and words. Journal of Experimental Psychology: General, 1976, 105, 378-402.

Coltheart, M., Hull, E., \& Slater, D. Sex differences in imagery and reading. Nature, 1975, 253, 438-440. 
DeLl, G. S. Slips of the mind. In M. Paradis (Ed.), The fourth Lacus forum. Columbia, S.C: Hornbeam Press, 1977.

Kolers, P. A., \& Smythe, W. E. Images, symbols, and skills. Canadian Journal of Psychology, 1979, 33, 158-184.

Kosslyn, S. M. Image and mind. Cambridge, Mass: Harvard University Press, 1980.

Landauer, T. K. Rate of implicit speech. Perceptual and Motor Skills, 1962, 15, 646.

MacKay, D. C. The problem of rehearsal or mental practice. Journal of Motor Behavior, 1981, 13, 274-285.

Mandler, G., \& DeAn, P. J. Seriation: Development of serial order in free recall. Journal of Experimental Psychology, 1969, 81, 207-215.

Meudel., P. R. Retrieval and representation in long-term memory. Psychonomic Science, 1971, 23, 295-296.

Paivio, A. Imagery and verbal processes. Toronto: Holt, Rinehart \& Winston, 1971.

Shepard, R. N. Learning and recall as organization and search. Journal of Verbal Learning and Verbal Behavior, 1966, 5, 201204.

Smith, M. C., \& MAgee, L. E. Tracing the time course of pictureword processing. Journal of Experimental Psychology: General, 1980, 109, 373-392.

Weber, R. J., \& BACH, M. Visual and speech imagery. British Journal of Psychology, 1969, 60, 199-202.
Weber, R. J., \& Castleman, J. The time it takes to imagine. Perception \& Psychophysics, 1970, 8, 165-168.

Weber, R. J., \& HaRnish, R. Visual imagery for words: The Hebb test. Journal of Experimental Psychology, 1974, 102, 409-414.

Weber, R. J., Kelley, J., \& Litrte, S. Is visual image sequencing under verbal control? Journal of Experimental Psychology, $1972,96,354-362$.

\section{NOTE}

1. Although the terms verbal (speech) and visual imagery appear ambiguous in that they can refer either to content or processing mode, colloquial use seems to respect the apparent correlation as well. As used in the present paper, verbal (speech) and visual imagery will refer to auditory and visual modes of imaginal processing, respectively. In reference to the specific use of a processing mode in the mental rehearsal task, the terms implicit verbal (visual) rehearsal will be used.

(Received for publication June 23, 1981; revision accepted February 10, 1982.) 